\title{
Partitions with Parts in a Finite Set and with Parts Outside a Finite Set
}

\author{
Gert Almkvist
}

\section{CONTENTS}

1. Introduction

2. Partitions into Parts in a Finite Set

3. Partitions with Parts Outside a Finite Set

References

2000 AMS Subject Classification: Primary 11P81;

Secondary 05A17, 11B34

Keywords: Partitions, asymptotics of partitions, additive number theory
Exact formulas for the number of partitions with parts in a finite set are proved without the use of partial fractions. Corresponding formulas with parts outside of a finite set are found. Several numerical examples are given.

\section{INTRODUCTION}

In a recent paper, M. B. Nathanson [Nathanson] finds the highest order term of the asymptotic formula for the number of partitions with parts in a finite set. This is done without referring to the partial fraction expansion of the generating function. In this paper, we essentially find the entire asymptotic formula without using partial fractions. This is done by the "metaphysical method" described in [Almkvist 98]. There the asymptotic formula was found using Fourier transformations of distributions in a rather obscure way. But when the generating function is rational, it is shown here that the method is perfectly sound. This is based on the following result:

\section{Lemma 1.1.}

$$
\operatorname{Res}\left(\frac{e^{n z}}{\left(1-e^{-z}\right)^{k}}, z=0\right)=\left(\begin{array}{c}
n+k-1 \\
k-1
\end{array}\right)
$$

In the second part of the paper, we consider partitions with parts outside a finite set. This is a complement of the situation in the first part. If

$$
A=\left\{a_{1}, \ldots, a_{k}\right\}
$$

is the finite set and $p(A, n)=$ number of partitions of $n$ with parts in $A$, then we have the generating function

$$
f_{A}(x)=\prod_{A}\left(1-x^{a}\right)^{-1}=\sum_{n=0}^{\infty} p(A, n) x^{n}
$$

(c) A K Peters, Ltd 1058-6458/2001 $\$ 0.50$ per page Experimental Mathematics 11:4, page 449 
Let $\widetilde{p}(A, n)=$ the number of partitions of $n$ with parts outside $A$, then we have

$$
\widetilde{f}_{A}(x)=\prod_{j \notin A}\left(1-x^{j}\right)^{-1}=\frac{F(x)}{f_{A}(x)}=\sum_{n=0}^{\infty} \widetilde{p}(A, n) x^{n}
$$

where

$$
F(x)=\prod_{j=0}^{\infty}\left(1-x^{j}\right)^{-1}=\sum_{n=0}^{\infty} p(n) x^{n}
$$

is the generating function of ordinary partitions $p(n)$. Since the expansions of $F(x)$ and $f_{A}(x)$ near $x=1$ are known, we also know the expansion of $\widetilde{f}_{A}(x)$ near $x=1$. This gives the first term in the asymptotic formula for $\widetilde{p}(A, n)$. We also do the same thing for $x$ near $x=-1$ to get the second term. Since $\widetilde{f}_{A}(x)$ is not rational, we do not have a proof, but numerically the approximation is excellent if $n$ is not too close to the largest number in $A$.

\section{PARTITIONS INTO PARTS IN A FINITE SET}

We start by proving that the "metaphysical method" is correct when the generating function is rational.

Theorem 2.1. Let $f(x)$ be a rational function with expansion

$$
f(x)=\sum_{n=0}^{\infty} c_{n} x^{n} .
$$

Let $x=\alpha$ be a pole of $f(x)$ of order $k$. Assume that

$$
f\left(\alpha e^{-t}\right)=\sum_{j=1}^{k} a_{j} t^{-j}+\sum_{j=0}^{\infty} b_{j} t^{j}
$$

when $t \rightarrow 0+$. Then the pole $\alpha$ gives rise to a term

$$
\alpha^{-n} \sum_{j=1}^{k} a_{j} \frac{n^{j-1}}{(j-1) !}
$$

in the asymptotic expansion of $c_{n}$ as $n \rightarrow \infty$.

Proof: It is enough to prove the theorem for

$$
\begin{aligned}
f(x)= & \frac{1}{(x-\alpha)^{k}}=\frac{(-1)^{k}}{\alpha^{k}} \frac{1}{\left(1-\frac{x}{\alpha}\right)^{k}} \\
& \frac{(-1)^{k}}{\alpha^{k}} \sum_{n=0}^{\infty}\left(\begin{array}{c}
n+k-1 \\
k-1
\end{array}\right) \alpha^{-n} x^{n} .
\end{aligned}
$$

Now

$$
f\left(\alpha e^{-t}\right)=\frac{(-1)^{k}}{\alpha^{k}} \frac{1}{\left(1-e^{-t}\right)^{k}} .
$$

Assume that

$$
\frac{1}{\left(1-e^{-t}\right)^{k}}=\sum_{j=1}^{k} d_{j} t^{-j}+O(1)
$$

We have to show that

$$
\sum_{j=1}^{k} d_{j} \frac{n^{j-1}}{(j-1) !}=\left(\begin{array}{c}
n+k-1 \\
k-1
\end{array}\right)
$$

Consider

$$
\frac{e^{n t}}{\left(1-e^{-t}\right)^{k}}=\sum_{i=0}^{\infty} \frac{n^{i} t^{i}}{i !}\left(\sum_{j=1}^{k} d_{j} t^{-j}+O(1)\right) .
$$

The coefficient of $t^{-1}$ of the right-hand side is

$$
\sum_{j=1}^{k} d_{j} \frac{n^{j-1}}{(j-1) !}
$$

so we are done if we can prove the following lemma:

Lemma 2.2. We have

$$
\operatorname{Res}\left(\frac{e^{n z}}{\left(1-e^{-z}\right)^{k}}, z=0\right)=\left(\begin{array}{c}
n+k-1 \\
k-1
\end{array}\right)
$$

Proof of Lemma 2.2: Let

$$
c(k, n)=\operatorname{Res}\left(\frac{e^{n z}}{\left(1-e^{-z}\right)^{k}}, z=0\right) .
$$

Then

SO

$$
\frac{e^{n z}}{\left(1-e^{-z}\right)^{k}}=\frac{e^{n z}}{\left(1-e^{-z}\right)^{k-1}}+\frac{e^{(n-1) z}}{\left(1-e^{-z}\right)^{k}},
$$

$$
c(k, n)=c(k-1, n)+c(k, n-1) .
$$

Clearly

$$
c(1, n)=1 \text { for all } n
$$

But

$$
\begin{aligned}
& \frac{1}{\left(1-e^{-z}\right)^{k-1}}-\frac{1}{\left(1-e^{-z}\right)^{k}} \\
& =-\frac{e^{-z}}{\left(1-e^{-z}\right)^{k}}=\frac{1}{k-1} \frac{d}{d z} \frac{1}{\left(1-e^{-z}\right)^{k-1}}
\end{aligned}
$$

has residue 0 . Hence

$$
c(k, 0)=c(k-1,0)=1 \text { for all } k .
$$

Now we do induction on both $k$ and $n$. Then

$$
c(k, n)=\left(\begin{array}{c}
n+k-2 \\
k-2
\end{array}\right)+\left(\begin{array}{c}
n+k-1 \\
k-1
\end{array}\right) .
$$


In order to state our next result, we introduce the following notation. Let

$$
\prod_{j=1}^{k} \frac{x_{j} t / 2}{\sinh \left(x_{j} t / 2\right)}=\sum_{m=0}^{\infty} \sigma_{m}\left(x_{1}, \ldots, x_{k}\right) t^{m}
$$

where $\sigma_{m}$ is a certain symmetric function (polynomial) of $x_{1}, \ldots, x_{k}$ similar to those introduced by Hirzebruch in topology. Clearly $\sigma_{0}=1$ and $\sigma_{m}=0$ if $m$ is odd. Let $A=\left\{a_{1}, \ldots, a_{k}\right\}$ be a set of positive integers without a common factor. Then

$$
f_{A}(x)=\prod_{A}\left(1-x^{a}\right)^{-1}=\sum_{n=0}^{\infty} p(A, n) x^{n} .
$$

Theorem 2.3. Let

$$
\xi=n+\frac{1}{2} \sum_{j=1}^{k} a_{j}
$$

Then the first approximation of $p(A, n)$, coming from the pole $x=1$ of $f(x)$ is

$$
\Phi_{1}(n)=\frac{1}{\prod_{A} a} \sum_{j=0}^{k-1} \sigma_{j}\left(a_{1}, \ldots, a_{k}\right) \frac{\xi^{k-j-1}}{(k-j-1) !} .
$$

Proof: We have

$$
\begin{aligned}
f\left(e^{-t}\right) & =\prod_{A}\left(1-e^{-a t}\right)^{-1} \\
& =\exp \left(\frac{t}{2} \sum_{A} a\right) \prod_{A} \frac{1}{\sinh (a t / 2)} \\
& =\frac{\exp \left(\frac{t}{2} \sum a\right) t^{-k}}{\prod a} \prod_{A} \frac{a t / 2}{\sinh (a t / 2)} \\
& =\frac{\exp \left(\frac{t}{2} \sum a\right)}{\prod a} \sum_{j} \sigma_{j}\left(a_{1}, \ldots, a_{k}\right) t^{-(k-j)} .
\end{aligned}
$$

The exponential $\exp \left(\frac{t}{2} \sum a\right)$ means just a translation (Taylor's theorem):

$$
n \longmapsto n+\frac{1}{2} \sum a=\xi .
$$

Thus the pole $x=1$ gives the contribution

$$
\Phi_{1}(n)=\frac{1}{\prod a} \sum_{j=0}^{k-1} \sigma_{j}\left(a_{1}, \ldots, a_{k}\right) \frac{\xi^{k-j-1}}{(k-j-1) !}
$$

to $p(A, n)$.
Example 2.4. (Partitions into primes.) Let

$$
A=\{2,3,5,7,11,13,17\}
$$

and

$$
f(x)=\prod_{a=2,3, . ., 17}\left(1-x^{a}\right)^{-1}=\sum_{n=0}^{\infty} c_{n} x^{n} .
$$

Then

$\prod_{A} \frac{a t / 2}{\sinh (a t / 2)}=1-\frac{111}{4} t^{2}+\frac{25807}{60} t^{4}-\frac{301359641}{60480} t^{6}+\ldots$ gives with $k=7$ and $\xi=n+\frac{1}{2} \sum a=n+29$

$\Phi_{1}(n)=\frac{1}{510510}\left\{\frac{\xi^{6}}{720}-\frac{37}{32} \xi^{4}+\frac{25807}{60} \xi^{2}-\frac{301359641}{60480}\right\}$.

If $n=10000$, then

$$
c_{10000}=2768250858256217
$$

while

$$
\Phi_{1}(10000)=2768250858256217.247 .
$$

Even better is

$$
c_{999}=3208335231
$$

with

$$
\Phi_{1}(999)=3208335231.00863 .
$$

This example is rather special since the $a_{j}: \mathrm{s}$ are pairwise coprime. In general, if

$$
f(x)=\frac{1}{1-x^{p}} \prod_{B}\left(1-x^{b}\right)^{-1}
$$

where $(p, b)=1$ for all $b \in B$, we will get a contribution

$$
\Phi_{p}(n)=\frac{1}{p} \sum_{\alpha} \frac{\alpha^{-n}}{\prod_{B}\left(1-\alpha^{b}\right)}
$$

where the sum runs over the nontrivial $p$-th roots of unity. In our example, we will get

$$
\Phi_{2}(n)=\frac{(-1)^{n}}{128} \text {. }
$$

If $p=3$, then

$$
(1-\alpha)\left(1-\alpha^{2}\right)=2-\left(\alpha+\alpha^{2}\right)=3
$$

so if $n=10000$, then

$$
\begin{aligned}
\Phi_{3} & =\frac{1}{3} \sum_{\alpha} \frac{\alpha^{-10000}}{\left(1-\alpha^{2}\right)\left(1-\alpha^{5}\right)\left(1-\alpha^{7}\right)\left(1-\alpha^{11}\right)\left(1-\alpha^{13}\right)\left(1-\alpha^{17}\right)} \\
& =\frac{1}{3} \sum_{\alpha} \frac{\alpha^{-1}}{(1-\alpha)^{2}\left(1-\alpha^{2}\right)^{4}}=\frac{1}{3^{5}} \sum \alpha^{2}(1-\alpha)^{2} \\
& =\frac{1}{3^{5}} \sum\left(-2+\alpha+\alpha^{2}\right)=\frac{1}{3^{5}}(-4-1-1)=-\frac{2}{81} .
\end{aligned}
$$


Similarly one finds

$$
\begin{aligned}
\Phi_{5} & =\frac{2}{25} \\
\Phi_{7} & =-\frac{5}{49} \\
\Phi_{11} & =-\frac{1}{11} \\
\Phi_{13} & =0 ; \\
\Phi_{17} & =-\frac{2}{17}
\end{aligned}
$$

One checks that

$$
\Phi_{1}+\Phi_{2}+\Phi_{3}+\Phi_{5}+\Phi_{7}+\Phi_{11}+\Phi_{13}+\Phi_{17}=c_{10000} .
$$

Example 2.5. (Partitions into Fibonacci numbers.) Let

$$
F_{1}=1, F_{2}=2, F_{n}=F_{n-1}+F_{n-2}
$$

be the Fibonacci numbers. Consider

$$
f(x)=\prod_{j=1}^{10}\left(1-x^{F_{j}}\right)^{-1}=\sum_{n=0}^{\infty} c_{n} x^{n} .
$$

Then we get the first approximation as before

$$
\begin{aligned}
\Phi_{1}(n)= & \frac{1}{r}\left\{\frac{\xi^{9}}{362880}-\frac{2563}{24192} \xi^{7}+\frac{322678417}{230400} \xi^{5}\right. \\
& \left.-\frac{90086094264}{129024} \xi^{3}+\frac{617952808976135603}{66355200} \xi\right\}
\end{aligned}
$$

where

$$
r=2 \cdot 3 \cdot 5 \cdot 8 \cdot 13 \cdot 21 \cdot 34 \cdot 55 \cdot 89
$$

and

$$
\xi=n+\frac{1}{2} \sum_{j=1}^{10} F_{j}=n+\frac{231}{2} .
$$

If $n=1000$, then

$$
c_{1000}=655200997428
$$

while

$$
\Phi_{1}(1000)=655200997007.243
$$

so the error is -420.75689 . We compute the second term coming from $x=-1$. Thus

$f\left(-e^{-t}\right)=\frac{1}{69632} t^{-3}+\frac{231}{139264} t^{-2}+\frac{20681}{278528} t^{-1}+\ldots$

giving

$$
\Phi_{2}(n)=(-1)^{n}\left\{\frac{1}{69632} \frac{n^{2}}{2}+\frac{231}{139264} n+\frac{20681}{278528}\right\} .
$$

In particular

$$
\Phi_{2}(1000)=\frac{2482681}{278528}=8.913577
$$

which is much smaller than the error -420.756 . So, what is going on? We compute the other terms as well. Let $\Phi_{k}$ denote the contributions from the $k$ :th roots of unity $\neq 1$ that have not been treated before. We get with $\alpha=\exp (2 \pi i / 3)$

$$
f(\exp (2 \pi i / 3-t))=\frac{1}{5103} t^{-2}+\left(\frac{11}{486}+\frac{i \sqrt{3}}{30618}\right) t^{-1}+\ldots
$$

and

$$
\begin{gathered}
\Phi_{3}(1000)=\frac{\alpha^{-1000}+\alpha^{1000}}{5103} 1000+\frac{11}{486}\left(\alpha^{-1000}+\alpha^{1000}\right) \\
+\frac{i \sqrt{3}}{30618}\left(\alpha^{-1000}-\alpha^{1000}\right)-\frac{1115}{5103}
\end{gathered}
$$

Now let $\alpha=\exp (2 \pi i / 5)$. Then

$$
\sum_{j=1}^{4} \alpha^{-1000 j} f\left(\alpha^{j} e^{-t}\right)=\frac{1}{11 \cdot 5^{4}}\left(t^{-2}+199 t^{-1}+\ldots\right)
$$

and

$$
\Phi_{5}(1000)=\frac{1}{11 \cdot 5^{4}}(1000+199)=\frac{1199}{11 \cdot 5^{4}} .
$$

Further computations using Maple give

$$
\begin{aligned}
\Phi_{4} & =-\frac{1}{256} \\
\Phi_{8} & =-\frac{1}{16} \\
\Phi_{13} & =\frac{3}{13} ; \\
\Phi_{21} & =-\frac{690}{147} \\
\Phi_{34} & =\frac{172}{17} ; \\
\Phi_{55} & =\frac{1382}{11} ; \\
\Phi_{89} & =\frac{24979}{89} .
\end{aligned}
$$

Hence $\Phi_{89}$ is the largest term! This depends on the fact that

$$
(1-\alpha)\left(1-\alpha^{2}\right)\left(1-\alpha^{3}\right) \cdots
$$

is very small when

$$
\alpha=\exp (2 \pi i / 89)
$$

When $n$ becomes large, $\Phi_{2}(n)$ will of course dominate. 
Remark 2.6. Partions into all Fibonacci numbers is a much more difficult problem. One needs to study the Fibonacci $\zeta$-function

$$
\zeta_{F i b}(s)=\sum_{n=1}^{\infty} \frac{1}{F_{n}^{s}}
$$

In particular one needs the values $\zeta_{F i b}(0)$ and $\zeta_{F i b}^{\prime}(0)$, etc.

Example 2.7. (Partitions into at most $\boldsymbol{r}$ parts.) Let $p(r, n)=$ the number of partitions of $n$ into parts $\leq r$ $=$ the number of partitions of $n$ into at most $r$ parts. We have the generating function

$$
f_{r}(x)=\prod_{j=1}^{r}\left(1-x^{j}\right)^{-1}=\sum_{n=0}^{\infty} p(r, n) x^{n}
$$

Then

$$
\begin{aligned}
\prod_{j=1}^{r} \frac{j t / 2}{\sinh (j t / 2)} & =\exp \left(-\sum_{j=1}^{r} \log \left(\frac{\sinh (j t / 2)}{j t / 2}\right)\right) \\
& =\exp \left(-\sum_{j=1}^{r} \sum_{k=1}^{\infty} \frac{B_{2 k} j^{2 k} t^{2 k}}{2 k(2 k) !}\right) \\
& =\exp \left(-\sum_{k=1}^{\infty} \frac{B_{2 k} B_{2 k+1}(r+1)}{2 k(2 k+1) !} t^{2 k}\right) .
\end{aligned}
$$

Hence

$f_{r}\left(e^{-t}\right)=\frac{t^{-r}}{r !} \exp \left\{\frac{r(r+1)}{4} t-\sum_{k=1}^{\infty} \frac{B_{2 k} B_{2 k+1}(r+1)}{2 k(2 k+1) !} t^{2 k}\right\}$ and we get the first approximation of $p(r, n)$ with $\xi=$ $n+r(r+1) / 4$

$$
\Phi_{1}(n)=\frac{1}{r !} \exp \left\{-\sum_{k=1}^{\infty} \frac{B_{2 k} B_{2 k+1}(r+1)}{2 k(2 k+1) !} D^{2 k}\right\} \frac{\xi^{r-1}}{(r-1) !}
$$

where

$$
D=\frac{d}{d \xi}
$$

If $r=11$, we get

$$
\begin{aligned}
\Phi_{1}(11, n)= & \frac{1}{11 ! \cdot 10 !}\left\{\xi^{10}-\frac{3795}{2} \xi^{8}+1190112 \xi^{6}\right. \\
& -\frac{567075465}{2} \xi^{4}+\frac{43143425875}{2} \xi^{2} \\
& \left.-\frac{10254376477121}{44}\right\} .
\end{aligned}
$$

We compute a few of the other terms:

$$
\Phi_{2}(11, n)=\frac{(-1)^{n}}{5 ! \cdot 4 ! \cdot 2^{11}}\left\{\xi^{4}-539 \xi^{2}+\frac{821381}{30}\right\} ;
$$

$$
\begin{aligned}
& \Phi_{3}(11, n)=\frac{1}{2 \cdot 3^{8}}\left\{\left(\xi^{2}-\frac{277}{2}\right) \cos \frac{2 \pi n}{3}+\frac{4 \xi}{\sqrt{3}} \sin \frac{2 \pi n}{3}\right\} ; \\
& \Phi_{4}(11, n)=\frac{1}{2^{10}}\left\{\xi \cos \frac{2 \pi n}{4}+3 \sin \frac{2 \pi n}{4}\right\} ; \\
& \Phi_{5}(11, n)=\frac{1}{2 \cdot 5^{4}}\left\{\frac{\sin \left((2 n+1) \frac{\pi}{5}\right)}{\sin \left(\frac{\pi}{5}\right)}+\frac{\sin \left((2 n+1) \frac{2 \pi}{5}\right)}{\sin \left(\frac{2 \pi}{5}\right)}\right\} \xi \\
&+\frac{1}{4 \cdot 5^{4}}\left\{\begin{array}{c}
\frac{\cos \left((2 n+1) \frac{\pi}{5}\right)}{\sin \left(\frac{\pi}{5}\right)}\left(5 \cot \left(\frac{\pi}{5}\right)-2 \cot \left(\frac{2 \pi}{5}\right)\right) \\
+\frac{\cos \left((2 n+1) \frac{2 \pi}{5}\right)}{\sin \left(\frac{2 \pi}{5}\right)}\left(2 \cot \left(\frac{\pi}{5}\right)+5 \cot \left(\frac{2 \pi}{5}\right)\right)
\end{array}\right\} ; \\
& \Phi_{6}(11, n)=\frac{\cos \left(\frac{n \pi}{3}\right)}{108} .
\end{aligned}
$$

Numerically we consider $n=1000$. Then

$$
p(11,1000)=9534808348706751
$$

We find

$$
\begin{aligned}
& \Phi_{1}=9534808348513832.336 ; \\
& \Phi_{2}=192956.508 ; \\
& \Phi_{3}=-40.497 ; \\
& \Phi_{4}=1.009 ; \\
& \Phi_{5}=1.657 ; \\
& \Phi_{6}=-0.005 .
\end{aligned}
$$

It follows that

$\Phi_{1}+\Phi_{2}+\Phi_{3}+\Phi_{4}+\Phi_{5}+\Phi_{6}=9534808348706751.007$.

Remark 2.8. Since

$$
\prod_{j=1}^{r-1}\left(1-e^{-j t}\right)^{-1}=\left(1-e^{-r t}\right) \prod_{j=1}^{r}\left(1-e^{-j t}\right)^{-1},
$$

we have (using Taylor's formula),

$$
\Phi_{1}(r-1, n)=\Phi_{1}(r, n)-\Phi_{1}(r, n-r) .
$$

\section{PARTITIONS WITH PARTS OUTSIDE A FINITE SET}

Let $A=\left\{a_{1}, \ldots, a_{k}\right\}$ be a finite set of positive integers. Let $\widetilde{p}(A, n)$ be the number of partitions of $n$ into parts not contained in $A$. The generating function is

$$
f_{A}(x)=\sum_{n=0}^{\infty} \widetilde{p}(A, n) x^{n}=\prod_{j \notin A}\left(1-x^{j}\right)^{-1} .
$$


Let

$$
F(x)=\prod_{j=1}^{\infty}\left(1-x^{j}\right)^{-1}=\sum_{n=0}^{\infty} p(n) x^{n}
$$

be the generating function of ordinary partitions. Let

$$
f_{A}(x)=\prod_{j \in A}\left(1-x^{j}\right)^{-1} .
$$

Then

$$
\tilde{f}_{A}(x)=\frac{F(x)}{f_{A}(x)} .
$$

Now by Hardy-Ramanujan's expansion, we know that

$$
F\left(e^{-t}\right) \approx \sqrt{\frac{t}{2 \pi}} \exp \left\{\zeta(2) t^{-1}-\frac{t}{24}\right\}
$$

and we have

$$
f_{A}\left(e^{-t}\right)=\frac{\exp \left(\frac{t}{2} \sum a\right)}{\prod a} t^{-k} \prod_{A} \frac{a t / 2}{\sinh (a t / 2)} .
$$

It follows that

$$
\begin{aligned}
\tilde{f}_{A}\left(e^{-t}\right) \approx \prod & a \frac{\exp \left(-\frac{t}{2} \sum a\right)}{\sqrt{2 \pi}} t^{k+1 / 2} \\
& \prod_{A} \frac{\sinh (a t / 2)}{a t / 2} \exp \left\{\zeta(2) t^{-1}-\frac{t}{24}\right\}
\end{aligned}
$$

Now

$$
\exp \left\{-t\left(\frac{1}{24}+\frac{1}{2} \sum a\right)\right\}
$$

is just a translation and $t^{k}$ means $D^{k}$. Formally we have

$$
\begin{aligned}
t^{1 / 2} \exp \left(\zeta(2) t^{-1}\right) & =\sum_{j=0}^{\infty} \frac{\zeta(2)^{j}}{j !} t^{1 / 2-j} \longmapsto \sum_{j=0}^{\infty} \frac{\zeta(2)^{j} n^{j-3 / 2}}{j ! \Gamma(j-1 / 2)} \\
& =\frac{1}{\sqrt{\pi \zeta(2)}} D^{2} \sinh \sqrt{4 \zeta(2) n}
\end{aligned}
$$

where

$$
D=\frac{d}{d n} \text {. }
$$

This is essentially the first term in the HardyRamanujan-Rademacher formula for $p(n)$. So let

$$
\xi=n-\frac{1}{24}-\frac{1}{2} \sum a .
$$

Then $\widetilde{p}(A, n)$ is approximated by

$$
\Phi_{1}(A, n)=\frac{\prod a}{\pi \sqrt{2 \zeta(2)}} \prod_{A} \frac{\sinh (a D / 2)}{a D / 2} D^{k+2} \sinh \sqrt{4 \zeta(2) \xi}
$$

where

$$
D=\frac{d}{d \xi}
$$

\section{Example 3.1. (Prime numbers.)}

Let $A=\{2,3,5,7,11,13,17\}$ as before. Then

$$
\begin{aligned}
\prod_{a=2,3, \ldots, 17} \frac{\sinh (a t / 2)}{a t / 2}=1 & +\frac{111}{2} t^{2}+\frac{81587}{240} t^{4} \\
& +\frac{2344127}{945} t^{6}+\frac{54918639}{4480} t^{8}+\ldots
\end{aligned}
$$

Thus we get with

$$
\xi=n-\frac{1}{24}-29
$$

that

$$
\begin{aligned}
\Phi_{1}(n)= & \frac{510510}{\pi \sqrt{2 \zeta(2)}}\left(1+\frac{111}{2} D^{2}+\frac{81587}{240} D^{4}\right. \\
& \left.+\frac{2344127}{945} D^{6}+\ldots\right) D^{9} \sinh \sqrt{4 \zeta(2) \xi}
\end{aligned}
$$

where

$$
D=\frac{d}{d \xi}
$$

Let $n=1000$. Then

$$
\widetilde{p}(A, 1000)=517150962488772205827376618
$$

while 13 terms give

$$
\Phi_{1}(1000)=517150962488712171137899967.647
$$

so we get 13 correct digits out of 27 .

We will now give a similar formula for the second term coming from the singularity at $x=-1$. Assume that

$$
A=B \smile C
$$

where $B$ consists of the even numbers in $A$ and $C$ of the odd. Then

$$
\begin{aligned}
f_{A}\left(-e^{-t}\right) & =\prod_{B}\left(1-e^{-b t}\right)^{-1} \prod_{C}\left(1+e^{-c t}\right)^{-1} \\
& =\frac{\exp \left(\frac{t}{2} \sum a\right)}{\prod_{B} b} t^{-|B|} \prod_{B} \frac{b t / 2}{\sinh (b t / 2)} \prod_{C} \frac{1}{2 \cosh (c t / 2)} .
\end{aligned}
$$

Furthermore, we have for

$$
F(x)=\prod_{j=1}^{\infty}\left(1-x^{j}\right)^{-1}
$$

the functional equation

$$
F(-x)=\frac{F\left(x^{2}\right)^{3}}{F(x) F\left(x^{4}\right)}
$$


which implies

$$
F\left(-e^{-t}\right) \approx \sqrt{\frac{t}{\pi}} \exp \left\{\frac{\zeta(2)}{4} t^{-1}-\frac{t}{24}\right\}
$$

and hence

$$
\begin{aligned}
\widetilde{f}_{A}\left(-e^{-t}\right)= & \frac{F\left(-e^{-t}\right)}{f_{A}\left(-e^{-t}\right)}= \\
= & \sqrt{\frac{t}{\pi}} \exp \left\{\frac{\zeta(2)}{4} t^{-1}-\frac{t}{24}\right\} \\
& \prod_{B} b \exp \left\{-\frac{1}{2} \sum a\right\} t^{|B|} \prod_{B} \frac{\sinh (b t / 2)}{b t / 2} \\
& \prod_{C}(2 \cosh (c t / 2)) .
\end{aligned}
$$

We get

$$
\begin{aligned}
\Phi_{2}(n)= & \frac{2 \prod b}{\pi \sqrt{\zeta(2)}} \prod_{B} \frac{\sinh (b D / 2)}{b D / 2} \\
& \prod_{C}(2 \cosh (c D / 2)) D^{|B|+2} \sinh \sqrt{\zeta(2) \xi}
\end{aligned}
$$

where

$$
\xi=n-\frac{1}{24}-\frac{1}{2} \sum_{A} a
$$

and

$$
D=\frac{d}{d \xi}
$$

We apply this formula when $A=\{2,3,5,7,11,13,17\}$. Then we have

$$
\begin{aligned}
\frac{\sinh (2 t / 2)}{2 t / 2} \prod_{3,5, \ldots, 17}\left(2 \cosh (c t / 2)=64+\frac{15920}{3} t^{2}\right. \\
+\frac{2650928}{15} t^{4}+\frac{200234249}{63} t^{6}+\cdots
\end{aligned}
$$

and we get

$$
\begin{aligned}
\Phi_{2}(n)= & \frac{2 \cdot 2}{\pi \sqrt{\zeta(2)}}\left\{64+\frac{15920}{3} D^{2}+\frac{2650928}{15} D^{4}\right. \\
& \left.+\frac{200234249}{63} D^{6}+\cdots\right\} D^{3} \sinh \sqrt{\zeta(2) \xi}
\end{aligned}
$$

where

$$
\xi=n-\frac{1}{24}-29
$$

If $n=1000$, we get taking 7 terms

$$
\Phi_{2}(1000)=60034649795838.605
$$

and

$$
\begin{array}{r}
\Phi_{1}(1000)+\Phi_{2}(1000) \\
=517150962488772205787695806.252
\end{array}
$$

so we get 19 correct digits out of 27 .

Example 3.2. (Partitions with parts $\geq \boldsymbol{r}$.) $\quad$ Let $A=$ $\{1,2, \ldots, r-1\}$. Then $\widetilde{p}(A, n)=\widetilde{p}(r, n)=$ the number of partitions of $n$ into parts $\geq r$. We have the generating function

$$
\widetilde{f}_{r}(x)=\sum_{n=0}^{\infty} \widetilde{p}(r, n) x^{n}=\prod_{j=r}^{\infty}\left(1-x^{j}\right)^{-1}=\frac{F(x)}{f_{r-1}(x)}
$$

Using the computations made in Example 2.7, we get

$$
\begin{aligned}
\widetilde{f}_{r}\left(e^{-t}\right)= & \frac{(r-1) !}{\sqrt{2 \pi}} t^{r-1 / 2} \\
& \exp \left\{\zeta(2) t^{-1}-\frac{B_{2}(r) t}{4}+\sum_{j=1}^{\infty} \frac{B_{2 j} B_{2 j+1}(r)}{2 j(2 j+1) !} t^{2 j}\right\}
\end{aligned}
$$

and

$$
\begin{aligned}
\Phi_{1}(r, n) & =\frac{(r-1) !}{\sqrt{2 \pi}} \\
& \exp \left\{\sum_{j=1}^{\infty} \frac{B_{2 j} B_{2 j+1}(r)}{2 j(2 j+1) !} D^{2 j}\right\} D^{r+1} \sinh (\sqrt{4 \zeta(2) \xi}
\end{aligned}
$$

where

$$
\xi=n-\frac{B_{2}(r)}{4}
$$

and

$$
D=\frac{d}{d \xi}
$$

As a numerical example, we take $r=12$ and $n=1200$. Then

$$
\widetilde{p}(12,1200)=49001590791729816727884124
$$

and

$$
\Phi_{1}(12,1200)=49001590791729483844367842.13295 .
$$

We also compute

$$
\Phi_{2}(12,1200)=332870971879.70797
$$

giving

$$
\Phi_{1}+\Phi_{2}=49001590791729816715339721.84092
$$

so we get 18 correct digits out of 26 . In a forthcoming paper, the author will show how to compute higher-order terms and hence compute $\widetilde{p}(12,1200)$ to the last digit. 
As a further example, we take $r=50$ and $n=1000$. Then

$$
\widetilde{p}(50,1000)=21837887237787
$$

and

$$
\Phi_{1}(50,1000)=21837887223761 \ldots
$$

so we get 12 digits out of 17 . Here we stopped after 18 terms of $\Phi_{1}$, since taking more terms destroys the result. It is still surprising that we can use the formula when $r$ is that big $(r>\sqrt{n})$. But when $B_{2}(r)>n$, then $\xi$ is negative and the formula is not valid. Then $\widetilde{p}(r, n)$ can be computed in the following way: Take $r=100$ and $n=1000$. Then

$$
\begin{aligned}
\widetilde{p}(100,1000)= & \sum_{j=1}^{10} p(j, 1000-100 j) \\
= & 1+401+41184+1537801+23030612 \\
& +134851969+263659693 \\
& +114281808+3314203+1 \\
= & 540717673 .
\end{aligned}
$$

Remark 3.3. Maple computes $p(n)$ immediately with "numbpart." This makes it possible to compute $\widetilde{p}(A, n)$ very fast Let

$$
\prod_{A}\left(1-x^{a}\right)=\sum_{j} c_{j} x^{j}
$$

Then

$$
\widetilde{p}(A, n)=\sum_{j} c_{j} p(n-j)
$$

\section{REFERENCES}

[Almkvist 98] G. Almkvist. "Asymptotic Formulas and Generalized Dedekind Sums." Exp. Math. 7:4 (1998), 343359

[Canfield 97] E. R. Canfield. "From Recursions to Asymptotics: On Szekeres' Formula for the Number of Partitions." Electr.J. of Comb. 4 (1997), \#R6.

[Dixmier and Nicolas 90] J. Dixmier and J. L. Nicolas. "Partitions sans petit sommands." In A Tribute to Paul Erdös, edited by A. Baker, B. Bollobas, and A. Hajnal, pp. 121-152. Cambridge: Cambridge Univ. Press, 1990.

[Nathanson] M. B. Nathanson. "Partitions with Parts in a Finite Set." Proc. Amer. Math. Soc. 128 (2000), 12691273 .

[Nicolas and Sarközy 97] J. L. Nicolas and A. Sarközy. "On Two Partition Problems." Acta Math. Hungar. 77 (1997), 95-121.

[Szekeres 51] G. Szekeres. "An Asymptotic Formula in the Theory of Partitions." Quart. J. of Math. 2 (1951), 85108.

[Szekeres 53] G. Szekeres. "Some Asymptotic Formulae in the Theory of Partitions (II)." Quart. J. of Math. 4 (1953), 96-111.

Gert Almkvist, Institute of Algebraic Meditation, PL 500, 24333 Höör, Sweden (gert@maths.lth.se)

Received December 5, 2001; accepted in revised form May 13, 2002. 